\title{
Session 6: Infectious disease
}

\author{
Wednesday 10 May, 2006. Moderators: Roberto Burioni and Mirek Gorny
}

\section{[16.00-16.30]}

Characterization of a quaternary epitope present on the surface of intact HIV-1 virions recognized by a human mAb

Miroslaw K. Gorny ${ }^{1}$, Tetsuya Kimura ${ }^{1}$, Barbara Volsky $^{1}$, Kathy Revesz ${ }^{2}$, Constance Williams ${ }^{1}$, William J.Honnen ${ }^{3}$, Chavdar Krachmarov ${ }^{3}$, Abraham Pinter $^{3}$ and Susan Zolla.Pazner ${ }^{1,2}$

${ }^{I}$ New York University School of Medicine, New York, NY 10016,USA

${ }^{2}$ Veterans Affairs New York Harbor Healthcare System, New York, NY 10010, USA

${ }^{3}$ Public Health Research Institute, Newark, NJ 07103, USA

The generation of human monoclonal antibodies (mAbs) against HIV-1 has been based entirely on the selection of mAbs that bind either to infected cells or to peptides and proteins representing the viral envelope. These methods resulted in the production of many interesting mAbs, including some that neutralize virus; but they have failed to define all of the antigenic structures that exist on the surface of intact virus particles, especially those where quaternary protein interactions occur. In order to determine if Abs to the quaternary epitopes exist and antibodies directed against them can neutralize primary HIV isolates, binding assays were replaced with a neutralization assay to screen for Ab-producing cells.

MAb 2909 was produced via fusion of heteromyeloma cells with EBV-transformed peripheral blood mononuclear cells (PBMC) derived from an HIVinfected individual. To select the cells for fusion, culture supernatants from EBV-transformed PBMCs were screened using a single-cycle infectivity assay for neutralization of virus pseudotyped with envelope proteins of primary isolate SF162. The hybridoma cells were cloned using the limiting dilutions method and each step of cloning was monitored by the neutralization assay.

MAb 2909 possesses remarkably potent typespecific neutralizing activity for SF162. Binding studies revealed that 2909 can bind only to intact SF162 virions, but not to its soluble envelope pro- teins. The quaternary structure of the 2909 epitope was determined by experiments showing the binding of 2909 to the trimeric but not to monomeric proteins expressed on the surface of the virus pseudotyped with native and mutated SF162 envelope proteins, respectively. Binding of 2909 to intact virus was competed by mAbs against V2, V3 and the CD4binding domains, suggesting that these regions contribute either directly or indirectly to the epitope. The mAb 2909 was further mapped by examining its neutralizing activity against SF162 mutants bearing changes in the V3 and V1/V2 domains. The results demonstrated a considerable tolerance for sequence variation in the V3 region but quite restricted specificity for the V1/V2 of SF162 which would explain the type-specific character of mAb 2909. This unique $\mathrm{mAb}$ defines a complex epitope formed by a quaternary structure on the virus surface, and this $\mathrm{mAb}$ may provide a new direction in the search for novel epitopes that can be targeted by vaccines.

[16.30-17.00]

Phage display and dissection of human antibody response against persistant viruses

Roberto Burioni

University San Raffaele, Milan, Italy

Abstract not received.

[17.00-17.20]

Towards the development of human monoclonal antibodies (MoAbs) for neutralization of "Spanish" influenza virus: Generation of hemagglutinin-specific mouse MoAbs and their characterization using pseudotyped influenza viruses

Luis Martínez-Sobrido ${ }^{1}$, Glenn Marsh ${ }^{1}$, Terrence M. Tumpey $^{2}$, Chistopher Basler ${ }^{1}$, Antonio Nakouzi ${ }^{3}$, Arturo Casadevall ${ }^{3}$ and Adolfo García-Sastre ${ }^{1}$

${ }^{I}$ Department of Microbiology, Mount Sinai School of Medicine, One Gustave L. Levy Place, New York, NY 10029, USA 
${ }^{2}$ Southeast Poultry Research Laboratory, Agricultural Research Service, U.S. Department of Agriculture, Athens, GA, USA

${ }^{3}$ Department of Medicine, Albert Einstein College of Medicine, 1300 Morris Park Avenue, Bronx, NY 10461, USA

In the last century, we have been affected by three influenza virus pandemics: the "Spanish flu" (19181919), the "Asian flu"(1957-1958), and the "Hong Kong flu" (1968-1969). The "Spanish flu" was the most devastating pandemic resulting in approximately 500,000 deaths in the United States and 40 million worldwide. The 1918 influenza virus was unique with respect to its high lethality in 20-40 years old humans, a property which was not seen in the later influenza virus pandemics. Studies aimed to understand the pathogenesis and immunogenicity of the 1918 influenza virus have been hampered by the absence of viral isolates from 1918-19. However, the determination of the sequence of the 1918 virus genes from preserved human tissue together with the development of reverse genetics techniques to generate influenza virus from plasmid DNA has allowed the rescue of viruses containing different combination of the 1918 genes. Importantly, the hemagglutinin (HA) gene was responsible for increased virulence in mice, suggesting a possible role of this gene in the unusual high lethality of the virus in humans. Therefore, the generation of neutralizing human MoAbs against the 1918 HA protein will provide potential prophylactic and therapeutic reagents useful for passive immunization of people who might have been exposed to the 1918 virus, as a result of an unfortunate laboratory accident or of an intentional release of the reconstructed 1918 virus by a terrorist organization. As a first step towards this goal, we have generated mouse MoAbs against the HA of the 1918 influenza virus. In order to study the neutralizing properties of these MoAbs, we have generated pseudotyped influenza viruses containing the 1918 HA. These pseudotyped viruses only propagate in influenza HA expressing cell lines and can be studied under BSL-2 conditions. Some of the MoAbs were characterized at the molecular level by determining their heavy and light chain variable region sequences, a necessary step for engineering these molecules into mouse-human chimeric antibodies. The identification of MoAbs with neutralizing properties against 1918 HA pseudotyped viruses will be presented and the benefits of these studies to the biodefense program will be discussed.
[17.20-17.40]

Generation of anti-orthopoxvirus fully human Mabs derived from phage display single-chain antibodies

Nina Tikunova et al.

State Research Center of Virology \& Biotechnology, Koltsovo, Russia

Abstract not received.

[17.40-18.00]

In vitro and in vivo characterization of $\mathrm{Ab2}-3 \mathrm{H} 6$ variants directed against the HIV-neutralising monoclonal antibody $2 \mathrm{~F} 5$

R. Kunert, J.Gach, S. Preis, M. Löschel, R. Weik, B. Ferko, G. Stiegler, Brititte Gasser Diedhard Mattanovich and H. Katinger

Institute Of Applied Microbiology, Muthgasse 18, 1190 Vienna Austria

Tel.: +431360066595 ; E-mail: renate.kunert@

boku.ac.at

Introduction: The development of a vaccine against HIV is one of the biggest concerns of today's health care policy. Different formats of neutralizing HIV epitopes as well as DNA-MVA candidate vaccines were tried in clinical studies with only little success. In our approach we used the neutralizing $\mathrm{mAb} 2 \mathrm{~F} 5$ for immunization of BALB/c mize and induced a humoral immune response. The generated antiidiotypic antibody $3 \mathrm{H} 6$ belongs to the Ab2beta subtype and is directed to the paratope of the Ab1$2 \mathrm{~F} 5$, bearing an internal image of the antigen. The $\mathrm{Ab} 2 \beta, 3 \mathrm{H} 6$, can compete with the nominal antigen for binding to the idiotype. To verify the specificity of the antibody, we expressed the it in different formats and in different hosts to control the immunochemical properties.

Expression of hybridoma derived and recombinant 3 H6.

Hybridoma derived 3H6: 2F5 immunized mice were tested for their serum anti-2F5 titer and animals with a specific titer of at least 1:100,000 were used for fusion. According to standard protocols the spleen cells were fused with X63-Ag-8.653. After selection, screening and stabilization of hybridoma cell lines the anti 2F5 specific hybridoma cell line $3 \mathrm{H} 6$ was propagated and the monoclonal antibody was isolated from the the culture supernatant.

Recombinant $\mathrm{CHO}$ cells expressing humanized 3H6: A humanized version of $3 \mathrm{H} 6$ was expressed in recombinant $\mathrm{CHO}$ cell lines, where the constant re- 
gions of the hybridoma derived cDNA was exchanged for human IgG1 and human ckappa regions. A production cell line was developed that expressed hu3H6 in $\mathrm{mg} / \mathrm{l}$ amounts in the cell culture supernatant.

Recombinant $\mathrm{CHO}$ cells expressing humanized 3H6-Fab: The expression of humanized 3H6-Fab fragments in $\mathrm{CHO}$ cells was done by deletion of the Hinge- $\mathrm{CH} 2-\mathrm{CH} 3$ heavy chain constant region. The secreted Fab fragments were purified by anion exchange chromatography and size exclusion chromatography and immunochemical properties were investigated.

Recombinant Pichia Pastoris cells expressing humanized 3H6-Fab point mutation variants: To get more information about the binding characteristics of the 3H6-Fab to the 2F5 paratope we constructed different point mutations in the CDR3 loop of $3 \mathrm{H} 6$ heavy chain and expressed them in Pichia Pastoris. Prolins and Tyrosin residues of the CDR3 region were exchanged for Alanins and single clones were sequenced and expressed in culture flasks.

Biochemical and immunochemical characteriza- tion: Culture supernatants from cell culture and yeast fermentations were purified by ProteinA affinity columnes or anion exchange chromatography combined with size exclusion chromatography and quantified in IgG and Fab specific ELISA. SDS gel electrophoresis and Northern Blot was used for control of purity and specific binding to the Ab1-2F5. Binding properties were measured in ELISA competition assays of comparable $3 \mathrm{H} 6$ preparations and the mutational variants.

The immunological characterization was controlled by in vitro-neutralization assays and best preparations will be further used as antigens to introduce HIV-spezific Ab3.

[18.00-18.30]

Protective human monoclonal antibodies against Pseudomonas in the clinic

Alois Lang

Berna Biotech, Berne, Switzerland

Abstract not received. 